\title{
ASSOCIATION BETWEEN THE CATECHOL-O-METHYLTRANSFERASE Val158Met POLYMORPHISM WITH SUSCEPTIBILITY AND SEVERITY OF CARPAL TUNNEL SYNDROME
}

\author{
Erkol İnal E ${ }^{1, *}$, Eroğlu P2 , Görükmez $\mathrm{O}^{3}$, Özemri Sağ Ş ${ }^{4}$, Yakut T ${ }^{4}$
}

*Corresponding Author: Dr. Esra Erkol İnal, Department of Physical Medicine and Rehabilitation, Süleyman Demirel University, Faculty of Medicine, Afyon yolu, Çünür, Isparta, Turkey. Tel: +90-246-211-9280. GSM: +90-507-563-6511. Fax: +90-246-211-2830. E-mail: esraerkol@hotmail.com

\begin{abstract}
Carpal tunnel syndrome (CTS) is the most common entrapment neuropathy of the upper extremity. In this study, we aimed to clarify the relationships between the catechol-O-methyltransferase (COMT) gene Val158Met (rs4680) polymorphism and development, functional and clinical status of CTS. Ninetyfive women with electro diagnostically confirmed CTS and 95 healthy controls were enrolled in the study. The functional and clinical status of the patients was measured by the Turkish version of the Boston Questionnaire and intensity of pain related to the past 2 weeks was evaluated on a visual analog scale (VAS). The Val158Met polymorphism was determined using the polymerase chain reaction-restriction fragment length polymorphism (PCR-RFLP), method. We divided patients according to the genotypes of the Val158Met polymorphism as Val/Val, Val/Met and Met/Met. There were not any significant differences in terms of Val158Met polymorphisms between patients and healthy controls $(p>0.05)$. We also did not find any relationships between the Val158Met polymorphism and functional and clinical status of
\end{abstract}

\footnotetext{
${ }^{1}$ Department of Physical Medicine and Rehabilitation, Süleyman Demirel University, Faculty of Medicine, Çünür, Isparta, Turkey

${ }^{2}$ Department of Physical Medicine and Rehabilitation, Ankara Occupational Disease Hospital, Ankara, Turkey

${ }^{3}$ Department of Medical Genetics, Bursa Şevket Yilmaz Education and Research Hospital, Bursa, Turkey

${ }^{4}$ Department of Medical Genetics, Uludağ University, Faculty of Medicine, Bursa, Turkey
}

CTS ( $p>0.05)$. In conclusion, although we did not find any relationships between CTS and the Val158Met polymorphism, we could not generalize this result to the general population. Future studies are warranted to conclude precise associations.

Keywords: Boston questionnaire, Carpal tunnel syndrome (CTS), catechol-O-methyltransferase (COMT) gene, pain, Val158Met (rs4680) polymorphism.

\section{INTRODUCTION}

Carpal tunnel syndrome (CTS) is the most common entrapment neuropathy of the upper extremity [1]. Patients with CTS may have different pain sensations. [2]. There is rising interest in the genetic predisposition to the painful conditions as they may be helpful in explaining the different pain responses to the same painful stimuli [3]. Three single nucleotide polymorphisms (SNPs) were accepted to impact pain perception: catechol-O-methyltransferase (COMT) Val158Met (rs4680), brain derived neurotrophic factor Val66Met and $\mu$-opioid receptor $1 A 118 G[4,5]$.

The COMT is an enzyme that metabolizes catecholamines such as dopamine, norepinephrine or epinephrine and has been reported to participate in the pathogenesis of several neuropsychiatric disorders [6]. The COMT gene is one of the several genes taking part in nociceptive processing; however, its role remains controversial. The COMT gene Val158Met SNP leads to a substitution of valine with methionine at codon 158 on chromosome 22q11. This substitution results in differences in the COMT enzyme ac- 
tivity [7]. The presence of the valine allele results in high enzymatic activity, whereas the presence of the methionine allele is linked to low enzymatic activity [8]. The Met/Met genotype was linked to increased pain sensitivity because of low enzymatic activity that leads the accumulation of catecholamines, whereas the Val/Val genotype results in reduced pain sensitivity. In only one study was the COMT gene Val158Met SNP found not to be related to CTS development but was associated with increased perception of pain and higher disability scores [9]. However, this result is not conclusive. Therefore, we aimed to determine the associations between the COMT gene Val158Met SNP and clinical and functional status of CTS.

\section{MATERIALS AND METHODS}

Participants. Ninety-five patients with CTS and 95 age- and ethnicity-matched healthy controls were enrolled in this study. All the participants were women and housewives. Informed consent from the all participants were obtained before being admitted to the study. The study was approved by the local ethics committee.

Patients were excluded from this study if they had any of the following: having previously undergone surgery for CTS, any sensory or motor deficit in the ulnar nerve, multiple diagnosis of the upper extremities such as lateral epicondylitis or cervical radiculopathy, history of systemic disease that causes CTS such as diabetes mellitus or hypothyroidism, concomitant systemic musculoskeletal conditions such as rheumatoid arthritis or fibromyalgia, pregnancy, previous fracture of the bones of upper extremities, trauma of the neck, shoulder or upper extremities and any other neurologic diseases. The patients had to have at least four of the following to be enrolled to undergo electroneurography (ENG): pain and paresthesia in the median nerve distribution without extra median nerve territory symptoms for at least six months; increasing symptoms at night; positive Tinel sign; positive Phalen sign and self-reported hand strength deficits. The age, gender, body mass index (BMI), symptoms duration and dominant hand of the patients were recorded. Tinel and Phalen signs were noted as positive or negative.

Functional and clinical status linked to CTS was evaluated by the Turkish version of the Boston Questionnaire that consists of the symptom severity scale (SSS) and the functional status scale (FSS). The SSS and the FSS include 11 and eight questions, respectively, which are scored with one (mildest) to five (most severe) points. The overall score is the mean of 11 scores assessing pain severity, numbness and weakness at night and day and eight scores assessing the difficulty in performing common hand-related tasks. Higher scores indicate worse symptoms or dysfunction [10]. The intensity of hand or wrist pain in the last 2 weeks was rated on VAS as $\mathrm{cm}$ by the patients.

Electroneurography (ENG) was performed with a two-channel ENG system (Micromed S.p.A., Mogliano Veneto TV, Italy) by an experienced physician when the patient was sitting with her arm semi-flexed. Electroneurography comprised motor and antidromic sensory conduction velocities of the median and ulnar nerves. Severity of the ENG was also classified according to standardized guidelines of the American Association of Electrodiagnosis, the American Academy of Neurology and the American Academy of Physical Medicine and Rehabilitation [11]. If median nerve sensory conduction velocity is less than $40 \mathrm{~mm} / \mathrm{s}$ and median nerve distal motor latency is greater than $4.20 \mathrm{~ms}$, they were considered to be abnormal. Only patients with abnormal segmental comparative tests were classified to have minimal CTS, while individuals with abnormal median nerve sensory velocity conduction and normal distal motor latency were considered to have mild CTS. To have moderate CTS, ENG has to reveal both abnormal median nerve sensory velocity conduction and distal motor latency. The patients who have abnormal median nerve motor distal latency and do not have median nerve sensory response are accepted to have severe CTS. Sensory and motor nerve conduction studies of the ulnar nerve were performed in order to rule out ulnar nerve lesions.

Blood samples from both patient and control groups were collected in vacutainers containing EDTA as anticoagulant. We isolated DNA according to the procedures of the DNA isolation kit used (Gentra Puregene Blood Kit; Qiagen GmbH, Hilden, Germany) and samples were stored at $-20{ }^{\circ} \mathrm{C}$ until analyzed by polymerase chain reaction (PCR).

The COMT gene Val158Met SNP was determined using the PCR-RFLP (restriction fragment length polymorphism) method. For the COMT gene Val158Met SNP, forward 5'-CTC ATC ACC ATC GAG ATC AA-3' and reverse 5'-CCA GGT CTG ACA ACG GGT CA-3' primers were used [12]. The PCR primers for Val158Met were used to generate a 
$109 \mathrm{bp}$ PCR product containing the polymorphic sites. Polymerase chain reaction products were digested overnight with NlaIII at $37^{\circ} \mathrm{C}$ and analyzed on $4.0 \%$ agarose gels. The fragments used to discriminate each genotype were as follows: valine homozygotes (86 and $23 \mathrm{bp}), \mathrm{Val} / \mathrm{Met}$ heterozygotes $(86,68,23$ and $18 \mathrm{bp}$ ), and methionine homozygotes (68 and $18 \mathrm{bp}$ ).

Statistical Analysis. Data were analyzed using the Statistical Package for the Social Sciences (SPSS) software version 15.0 for Windows (SPSS Inc., Chicago, IL, USA). Mean and standard deviations (mean $\pm \mathrm{SD}$ ) were used for the presentation of continuous quantitative variables. Frequencies and percentages were used for categorical data. The one-way analysis of variance (ANOVA) test was used for parametric variables, and for non parametric variables, the Kruskal-Wallis test was used for comparisons among the three groups. For evaluation of categorical variables, a $\chi^{2}$ test and if needed, Fisher's exact $t$-test were used. Comparisons of genotype distribution and allele frequency between the groups were performed using a $\chi^{2}$ test. To determine whether the allele frequencies were stable within patients and controls, $\chi^{2}$ analysis of the Hardy-Weinberg equilibrium for the genotypes was conducted. A $p$ value of $<0.05$ was accepted as significant for all statistical analyses.

\section{RESULTS}

The ages of the patients and the healthy controls were $46.3 \pm 12.0$ and $46.8 \pm 11.3$ years, respectively $(p=0.775)$. No one showed minimal CTS, 47 women had mild CTS, 40 of the patients had moderate CTS, and the remaining eight had severe CTS. The mean duration of the symptoms was $31.5 \pm 36.6$ months, while the mean pain level relating to the last 2 weeks was $7.01 \pm 3.4 \mathrm{~cm}$ on VAS.

The Val158Met genotype distributions in women with CTS and healthy women significantly deviated from the Hardy-Weinberg equilibrium $(p<0.001$ for both). There was not a significant difference in the distribution of the genotypes and alleles of the COMT gene Val158Met SNP between women with CTS and healthy women $(p=0.46)$ (Table 1$)$. We also did not find any significant difference according to genotypes in terms of symptom duration, severity of pain and ENG, and Boston FSS and SSS (Table 2).

Table 1. Distribution of the Val158Met genotypes and alleles of the catechol-O-methyltransferase gene in women with carpal tunnel syndrome and healthy women.

\begin{tabular}{|l|c|c|c|}
\hline Parameters & Women with CTS $(\boldsymbol{n}=\mathbf{9 5})$ & Healthy Controls $(\boldsymbol{n}=\mathbf{9 5})$ & $\boldsymbol{p}$ Value \\
\hline Val158Met Polymorphism Genotype & & & \\
Met/Met & $5(5.3 \%)$ & $2(2.1 \%)$ & 0.460 \\
Val/Met & $60(63.2 \%)$ & $65(68.4 \%)$ & \\
Val/Val & $30(31.6 \%)$ & $28(29.5 \%)$ & \\
\hline Alleles & $70(41.9 \%)$ & $69(41.9 \%)$ & 0.915 \\
Met & $120(58.1 \%)$ & $121(58.1 \%)$ & \\
Val & & & \\
\hline
\end{tabular}

Val: valine; Met: methionine; CTS: carpal tunnel syndrome.

Table 2. Demographic and clinic features in women with the carpal tunnel syndrome according to the Val158Met polymorphism genotypes.

\begin{tabular}{|l|c|c|c|c|}
\hline Parameters & Met/Met & Val/Met & Val/Val & $p$ Value \\
\hline Age $($ years) & $40.7 \pm 12.3$ & $47.0 \pm 11.6$ & $46.3 \pm 11.5$ & 0.418 \\
\hline BMI $\left(\mathrm{kg} / \mathrm{cm}^{2}\right)$ & $27.5 \pm 5.6$ & $29.6 \pm 4.8$ & $29.4 \pm 5.5$ & 0.312 \\
\hline Symptom duration (months) & $54.0 \pm 84.2$ & $28.5 \pm 23.0$ & $33.8 \pm 46.5$ & 0.303 \\
\hline Severity of pain (cm) & $8.0 \pm 2.7$ & $7.4 \pm 3.2$ & $6.3 \pm 4.0$ & 0.392 \\
\hline Severity of ENG (mild/moderate/severe) & $2 / 2 / 1$ & $26 / 28 / 6$ & $19 / 10 / 1$ & 0.347 \\
\hline Tinel sign (N/P/PP) & $0 / 4 / 3$ & $3 / 21 / 14$ & $2 / 35 / 13$ & 0.572 \\
\hline Phalen sign (N/P/PP) & $0 / 5 / 3$ & $3 / 20 / 14$ & $2 / 35 / 13$ & 0.551 \\
\hline Boston FSS & $1.8 \pm 0.9$ & $2.5 \pm 3.0$ & $2.1 \pm 1.0$ & 0.530 \\
\hline Boston SSS & $2.8 \pm 1.4$ & $2.9 \pm 0.9$ & $2.8 \pm 0.9$ & 0.986 \\
\hline
\end{tabular}

CTS: carpal tunnel syndrome; Met: methionine; Val: valine; BMI: body mass index; ENG: electroneurography; N: bilateral negative; P: unilateral positive; PP: bilateral positive; Boston FSS: Boston functional status scale; Boston SSS: Boston symptom severity scale. 


\section{DISCUSSION}

Lower COMT gene activity that is linked to Met/ Met genotype, results in elevated levels of catecholamines, which stimulate $\beta 2$ adrenergic receptors in the peripheral and central nervous system $[13,14]$. The clinical status in CTS is generally thought be a result of the entrapment of the median nerve, nevertheless, current results revealed the association of the Met/Met genotype with higher pain sensitivity and disability in a number of chronic musculoskeletal disorders such as fibromyalgia [15], temporomandibular pain [16] and CTS [9]. However, our data suggested that there was no relationship between pain severity and disability and the COMT gene Val158Met SNP in the patients with CTS, similar to some previous studies investigating chronic musculoskeletal disorders [17] and neuropathic [18] and widespread pain [19]. These discrepancies may be due to the fact that a patient's SNP in a potential candidate gene does not act alone, but in interaction with environmental and ethnic factors to reveal personal manifestation in the disease course.

The COMT gene Val158Met SNP Met/Met genotype was found to be related with higher anxiety, depression and disability in fibromyalgia [20,21]. The COMT gene SNPs related to pain sensitivity such as Val158Met was found to be related with severity of fibromyalgia in Spanish patients, whereas there was no relation in Mexican patients [22]. The patients with the Met/Met genotype had worse clinical features in long-lasting low-back pain, sciatica and lumbar disc herniation $[23,24]$. Nevertheless, the exact role of the COMT gene Val158Met SNP remains controversial. Supporting all these results: gender, ethnicity and psychological state were accepted to interact with the genes in pain responses to painful conditions [5].

Similar to a recent study, we did not find any relationships between the COMT gene Val158Met SNP and development of CTS [9]. The patient population in the latter study also consisted of solely female patients similar to the present study.

There are several limitations in the present study. The population in this study includes only female patients and the participants were all from a secondary hospital. These conditions may limit the results to the general population. Moreover, only one SNP of the COMT gene was investigated in this study; therefore, we could not exclude the other genetic influences.

In conclusion, we found no relationships be- tween the Val158Met SNP, CTS and clinical outcomes. Genetic researches can help to offer special care to the patients; however, the results of the reports investigating the relationships between the COMT gene Val158Met SNP and pain perception in painful conditions are conflicting. New studies with a greater number of candidate genes to CTS and pain, in a larger population including both male and female patients from several hospitals and geographic regions, should be designed to establish precise relations.

Declaration of Interest. The authors report no conflicts of interest. The authors alone are responsible for the content and writing of this article.

\section{REFERENCES}

1. Bland JD. Carpal tunnel syndrome. BMJ. 2007; 335(7615): 343-346.

2. Zanette G, Cacciatori C, Tamburin S. Central sensitization in carpal tunnel syndrome with extraterritorial spread of sensory symptoms. Pain. 2010; 148(2): 227-236.

3. Vossen H, Kenis G, Rutten B, van Os J, Hermens H, Lousberg R. The genetic influence on the cortical processing of experimental pain and the moderating effect of pain status. PLoS One. 2010;5:e13641. doi: 10.1371/journal. pone. 0013641 .

4. Serý O, Hrazdilová O, Matalová E, Sevcík P. Pain research update from a genetic point of view. Pain Pract. 2005; 5(4): 341-348.

5. Kim H, Neubert JK, San Miguel A, Xu K, Krishnaraju RK, Iadarola MJ, et al. Genetic influence on variability in human acute experimental pain sensitivity associated with gender, ethnicity and psychological temperament. Pain. 2004; 109(3): 488-496.

6. Zhu BT. Catechol-O-Methyltransferase (COMT)-mediated methylation metabolism of endogenous bioactive catechols and modulation by endobiotics and xenobiotics: Importance in pathophysiology and pathogenesis. Curr Drug Metab. 2002; 3(3): 321-349.

7. Mänistö PT, Kaakkola S. Catechol-O-methyltransferase (COMT): Biochemistry, molecular biology, pharmacology, and clinical efficacy of the new selective COMT inhibitors. Pharmacol Rev. 1999; 51(4): 593-628. 
8. Chen J, Lipska BK, Halim N, Ma QD, Matsumoto $\mathrm{M}$, Melhem $\mathrm{S}$, et al. Functional analysis of genetic variation in catechol-O-methyltransferase (COMT): Effects on mRNA, protein, and enzyme activity in postmortem human brain. Am J Hum Genet. 2004; 75(5): 807-821.

9. Fernández-de-las-Peñas $\mathrm{C}$, Ambite-Quesada $\mathrm{S}$, Ortega-Santiago R, Martínez-Perez A, Díaz HF, Martínez-Martín J, et al. Catechol-O-methyltransferase Val158Met polymorphism is associated with pain and disability, but not widespread pressure pain sensitivity, in women with carpal tunnel syndrome. Pain Physician. 2013; 16(6): 591-600.

10. Sezgin M, Incel NA, Serhan S, Camdeviren H, As I, Erdoğan C. Assessment of symptom severity and functional status in patients with carpal tunnel syndrome: Reliability and functionality of the Turkish version of the Boston Questionnaire. Disabil Rehabil. 2006; 28(20): 1281-1285.

11. American Association of Electrodiagnostic Medicine, American Academy of Neurology, and American Academy of Physical Medicine and Rehabilitation. Practice parameter for electrodiagnostic studies in carpal tunnel syndrome: Summary statement. Muscle Nerve. 2002; 25(6): 918-922 (https://www.aanem.org/getmedia/ 95a3a91e-a196-4d1c-bbc9).

12. Albaugh MD, Harder VS, Althoff RR, Rettew DC, Ehli EA, Lengyel-Nelson T, et al. COMT Val158Met genotype as a risk factor for problem behaviors in youth. J Am Acad Child Adolesc Psychiatry. 2010; 49(8): 841-849.

13. Diatchenko L, Slade GD, Nackley AG, Bhalang $\mathrm{K}$, Sigurdsson A, Belfer I, et al. Genetic basis for individual variations in pain perception and the development of a chronic pain condition. Hum Mol Genet. 2005; 14(1): 135-143.

14. Segall SK, Maixner W, Belfer I, Wiltshire T, Seltzer Z, Diatchenko L. Janus molecule I: Dichotomous effects of COMT in neuropathic vs nociceptive pain modalities. CNS Neurol Disord Drug Targets. 2012; 11(3): 222-235.

15. Barbosa FR, Matsuda JB, Mazucato M, de Castro França S, Zingaretti SM, da Silva LM, et al. Influence of catechol-O-methyltransferase (COMT) gene polymorphisms in pain sensibility of Brazilian fibromyalgia patients. Rheumatol Int. 2012; 32(2): 427-430.
16. Smith SB, Maixner DW, Greenspan JD, Dubner R, Fillingim RB, Ohrbach R, et al. Potential genetic risk factors for chronic TMD: Genetic associations from the OP PERA case control study. J Pain. 2011; 12(11 Suppl): 92-101.

17. Hagen K, Pettersen E, Stovner LJ, Skorpen F, Zwart JA. No association between chronic musculoskeletal complaints and Val158Met polymorphism in the catechol-O-methyltransferase gene. The HUNT study. BMC Musculoskelet Disord. 2006; 7: 40.

18. Armero P, Muriel C, Santos J, Sanchez-Montero FJ, Rodríguez RE, González-Sarmiento R. COMT (Val158 Met) polymorphism is not associated to neuropathic pain in a Spanish population. Eur J Pain. 2005; 9(3): 229-232.

19. Nicholl BI, Holliday KL, Macfarlane GJ, Thomson W, Davies KA, O’Neill TW, et al.; European Male Ageing Study Group. No evidence for a role of the catechol-O-methyltransferase pain sensitivity haplotypes in chronic widespread pain. Ann Rheum Dis. 2010; 69(11): 2009-2012.

20. Cohen H, Neumann L, Glazer Y, Ebstein RP, Buskila D. The relationship between a common catechol-O-methyltransferase (COMT) polymorphism val(158)met and fibromyalgia. Clin Exp Rheumatol. 2009; 27(5 Suppl 56): 51-56.

21. Fernández-de-Las-Peñas $\mathrm{C}$, Ambite-Quesada $\mathrm{S}$, Gil-Crujera A, Cigarán-Méndez M, PecacobaPuente C. Catechol-O-methyltransferase Val158Met polymorphism influences anxiety, depression, and disability, but not pressure pain sensitivity, in women with fibromyalgia syndrome. J Pain. 2012; 13(11): 1068-1074.

22. Vargas-Alarcyn, JG, Fragoso M, Cruz-Robles D, Vargas A, Vargas A, Lao-Villadyniga JI, et al. Catechol-O-methyltransferase gene haplotypes in Mexican and Spanish patients with fibromyalgia. Arthritis Res Ther. 2007; 9(6): 110.

23. Jacobsen LM, Schistad EI, Storesund A, Pedersen LM, Rygh LJ, Røe C, et al. The COMT rs4680 Met allele contributes to long-lasting low back pain, sciatica and disability after lumbar disc herniation. Eur J Pain. 2012; 16(7): 1064-1069.

24. Omair A, Lie BA, Reikeras O, Holden M, Brox JI. Genetic contribution of catechol-O-methyltransferase variants in treatment outcome of low back pain: A prospective genetic association study. BMC Musculoskelet Disord. 2012; 13: 76. 
